\title{
Evaluation of Mobility Status after Inguinal Hernia Surgery
}

\author{
Egle Kubiliute $^{a}$ Linas Venclauskas $^{b} \quad$ Kristijonas Jasaitis $^{b} \quad$ Ernestas Margelis $^{b}$ \\ Mindaugas Kiudelis ${ }^{b}$ \\ ${ }^{a}$ Department of General Surgery, Lithuanian University of Health Sciences, Kaunas, Lithuania; \\ ${ }^{b}$ Department of Surgery, Lithuanian University of Health Sciences, Kaunas, Lithuania
}

\section{Keywords}

Inguinal hernia - Laparoscopic-endoscopic hernia repair - Open hernia repair · Pelvic function · Postoperative recovery

\section{Abstract}

Background: Many different studies have compared open and laparoscopic-endoscopic inguinal hernia repair techniques according to intraoperative and postoperative complications, recurrence rates, postoperative inguinal chronic pain, quality of life, and costs. Most of the researchers have compared these different inguinal hernia repair techniques using a visual analog scale, a short-form survey instrument, or patients' return-to-normal-activity time, but there is a lack of objective data concerning pelvic function recovery after these procedures. Aim: To evaluate and compare real hip and leg function recovery times after the application of different inguinal hernia repair techniques using hip and leg mobility, strength, and stability testing for the first time. Patients and Methods: This prospective nonrandomized clinical study included 33 male patients aged 18-75 years hospitalized for primary inguinal hernia repair surgery. The patients were divided into two groups: group 1 (Lichtenstein hernia repair) and group 2 (laparoscopic-endoscopic transabdominal preperitoneal/totally extraperitoneal hernia repair). The two groups were compared in terms of intraoperative and postoperative complications, postoperative recovery time, and hip and leg mobility, strength, and stability functional analysis on the first postoperative day as well as 1, 2, and 4 weeks after surgery. Results: $A$ total of 33 patients were included in the study: 13 in the open hernia repair group and 20 in the minimally invasive hernia repair group. There was no significant difference in early and late postoperative complications and recurrence rates. The surgery time and hospital stay were significantly shorter in the laparoscopic-endoscopic hernia repair group. All pelvic functions in the patients who underwent laparoscopic-endoscopic hernia repair recovered 2 or 3 weeks faster than after Lichtenstein repair. Conclusions: Hip and leg mobility, strength, and stability tests are useful to evaluate the recovery time after inguinal hernia repair and could be used as objective tools for estimating recovery after the application of other inguinal hernia repair techniques. Hip and leg mobility, strength, and stability recover faster after minimally invasive inguinal hernia repair. There is no significant difference between the groups in early and late postoperative complications or recurrence rates.

(c) 2019 S. Karger AG, Basel

\section{Introduction}

Inguinal hernia repair is one of the most commonly performed surgical procedures worldwide. About 20 million patients undergo inguinal hernia repair surgery every year. Mesh repair is the optimal choice for inguinal hernia repair, using either an open or a laparoscopicendoscopic technique [1].

Data from this paper have in part been presented at the 9th Congress of the Baltic Association of Surgeons (Klaipeda, Lithuania; May 10-12, 2018).

\section{KARGER}

() 2019 S. Karger AG, Basel 
Many studies have compared open with laparoscopicendoscopic hernia repair techniques according to intraoperative and postoperative complications, recurrence rates, postoperative pain, quality of life, and costs. The HerniaSurge Group analysis showed no significant difference in severe chronic pain and long-term recurrence rates between the two groups. Also, laparoscopic-endoscopic techniques have advantages in terms of early and late postoperative pain and recovery time [2].

The World Guidelines for Groin Hernia Management states that for male patients with primary unilateral inguinal hernia, a laparoscopic-endoscopic technique is recommended because of a lower incidence of postoperative pain and a reduction in the incidence of chronic pain, provided that a surgeon with specific and sufficient resources is available [1]. The European Hernia Society guidelines recommend that an endoscopic technique be considered if quick postoperative recovery is particularly important; from a socioeconomic perspective, an endoscopic procedure is also proposed for the active working population, especially in the case of bilateral hernias [3].

However, most researchers have compared open with laparoscopic-endoscopic hernia repair techniques using a visual analog scale, a short-form survey instrument, or patients' return-to-normal-activity time, but there is a lack of objective data concerning pelvic function recovery after these procedures [4-7]. We designed this clinical study to objectively evaluate and compare real recovery times after the application of different inguinal hernia repair techniques.

\section{Patients and Methods}

This prospective clinical study included 33 male patients aged 18-75 years hospitalized for primary inguinal hernia repair surgery. All the patients gave their written informed consent, and the study protocol was evaluated and approved by the Ethics Committee of the Lithuanian University of Health Sciences (protocol No. BEC-LSMUC(R)-26). The exclusion criteria were strangulated hernia, age older than 75 years, being in ASA class IV, and having neurological or psychiatric disorders. The included patients were divided into two groups: group 1 (Lichtenstein hernia repair) and group 2 (laparoscopic-endoscopic transabdominal preperitoneal/ totally extraperitoneal [TEP] hernia repair).

Three experienced surgeons performed all the open and laparoscopic-endoscopic operations. The two groups were compared in terms of intraoperative and postoperative complications, postoperative recovery time, and pelvic functional analysis.

Leg strength testing by dynamometer, mobility assessment by goniometer, and the FMS Y Balance Test were used to objectively evaluate pelvic function.

\section{Mobility Test}

1. The patient is lying on the back and one of his legs is passively bent through the hip joint until there is pain in the inguinal area. When the pain occurs, the angle of the folding leg is measured with the goniometer in the area of the greater trochanter (Fig. 1)

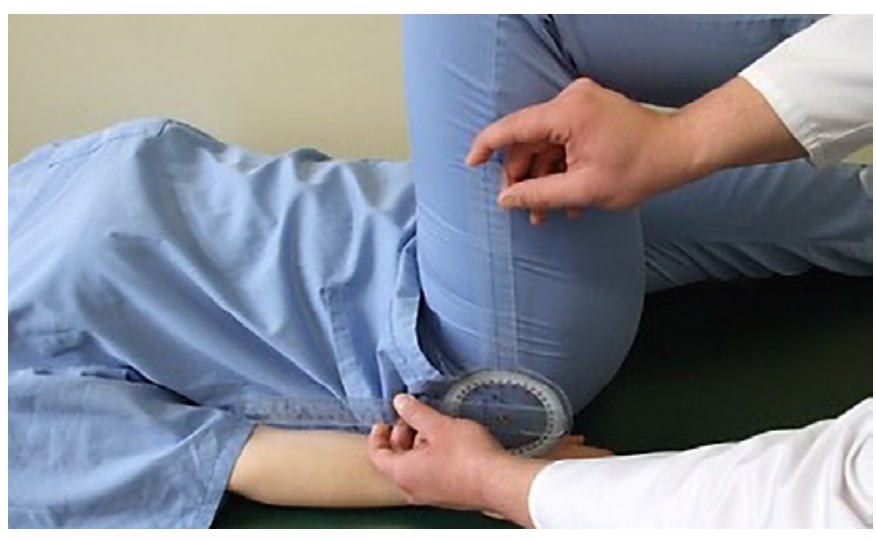

Fig. 1. Mobility test: flexion angle.

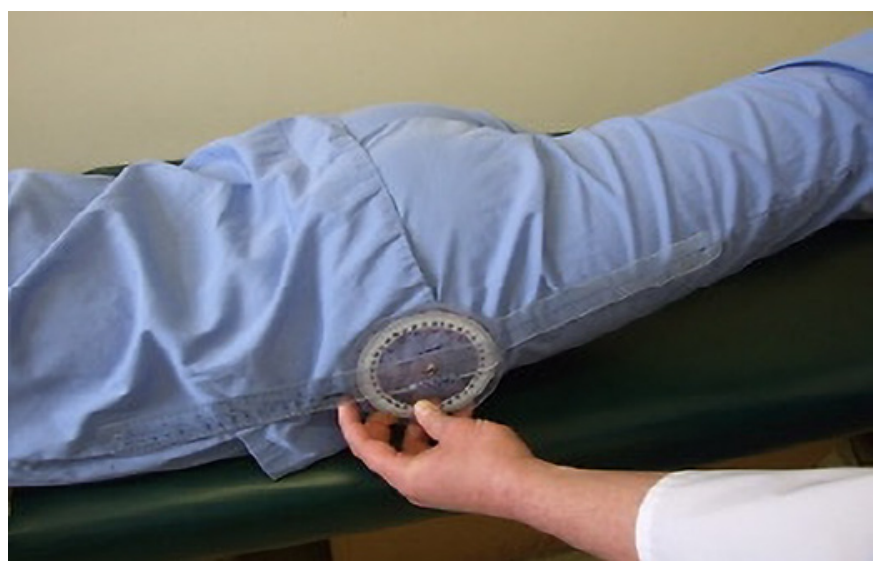

Fig. 2. Mobility test: extension angle.

2. The patient is lying on the belly and his leg is passively stretched through the hip joint until there is pain in the inguinal area. When the pain occurs, the angle of the stretching leg is measured with the goniometer in the area of the greater trochanter (Fig. 2)

3. The patient is lying on the back and his leg is passively adducted through the hip joint until there is pain in the inguinal area. When the pain occurs, the angle of the adducted leg is measured with the goniometer in the pubic area (Fig. 3)

4. Measurements are performed on both legs

\section{Strength Test}

1. The patient is lying on the back and he bends his leg actively through the hip joint until there is pain in the inguinal area. The researcher puts the dynamometer on the patella and creates resistance against the bending leg. When the pain occurs, strength is measured with the dynamometer (Fig. 4)

2. The patient is lying on the back and he adducts his leg actively through the hip joint. The researcher sets the dynamometer on the outer side of the knee and creates resistance against the adducting leg. When the pain occurs, strength is measured with the dynamometer (Fig. 5)

3. The patient is lying on the back and he abducts his leg actively through the hip joint until there is pain in the inguinal area. The researcher sets the dynamometer on the inner side of the knee and creates resistance against the abducting leg. When the pain occurs, strength is measured with the dynamometer (Fig. 6)

4. Measurements are performed on both legs 


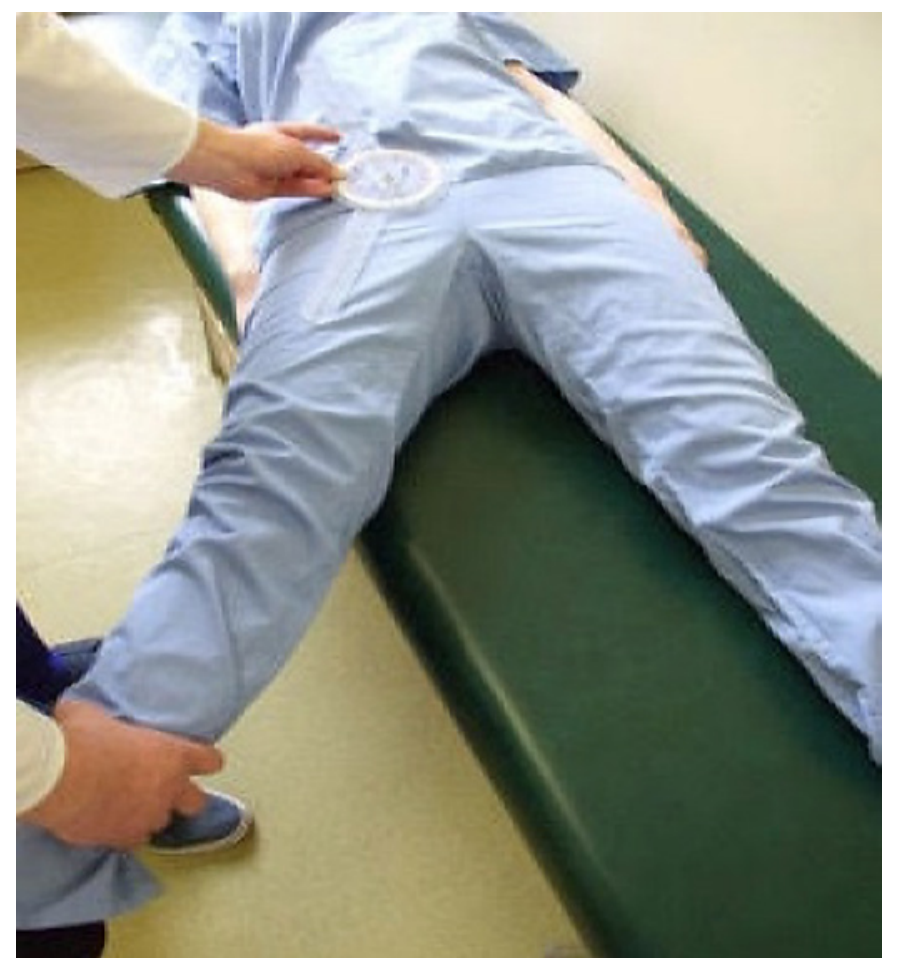

Fig. 3. Mobility test: adduction angle.

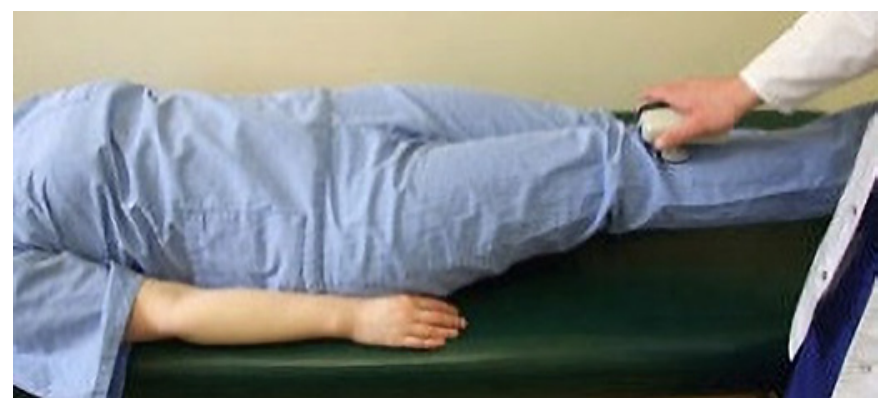

Fig. 4. Strength test: flexion strength.

Stability Test

1. The FMS Y Balance Test instrument is used. The patient is standing on one leg, pushing the instrument's block forward posteromedially and posterolaterally with the other leg as much as he can without feeling pain and without touching the ground with his feet. After every movement, the patient puts his feet on the ground. Measurements are repeated 3 times, and the best result is chosen (Fig. 7)

2. The result is considered the average of these 3 dimensions

\section{Time of Testing}

All tests were performed before the operation, on the first postoperative day, and 1,2, and 4 weeks after surgery.

\section{Statistical Analysis}

Fisher's exact test was used to compare the types of hernia, early and late postoperative complications, and recurrence rates of the two treatment groups. Patient age, hospital stay, and pelvic functional data changes were compared between the groups using the unpaired Mann-Whitney $U$ test. $p<0.05$ was considered significant.

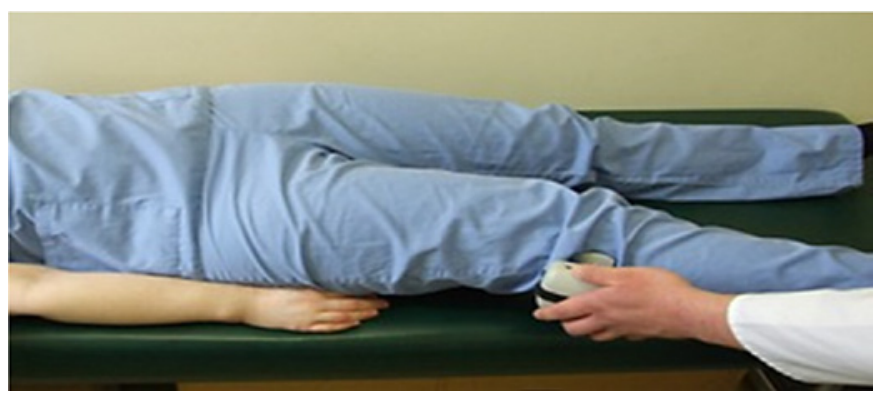

Fig. 5. Strength test: adduction strength.

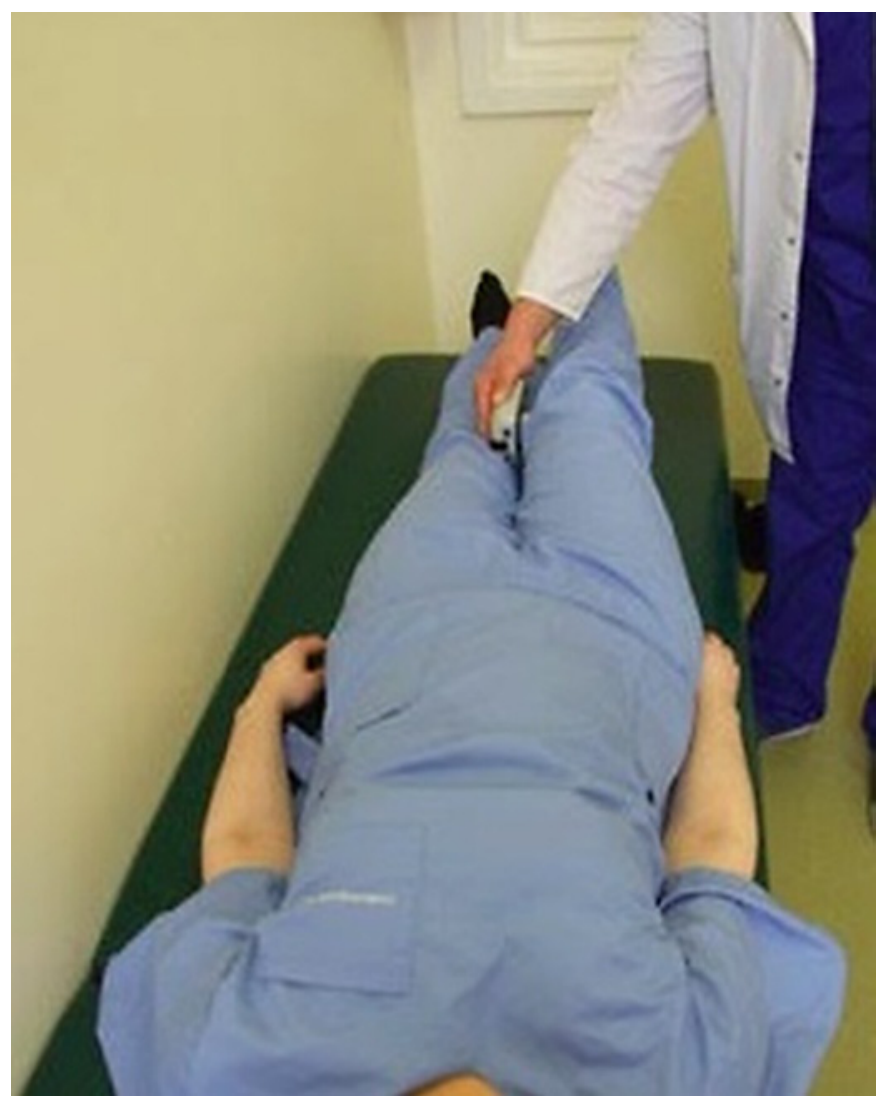

Fig. 6. Strength test: abduction strength.

Results

A total of 33 patients were included in the study; 13 underwent Lichtenstein hernia repair and 20 minimally invasive hernia repair. The general characteristics were similar in both groups, and no significant differences were observed in any of those data (Table 1). However, the surgery time was significantly shorter in the laparoscopic-endoscopic group, whereas the Lichtenstein group had a significantly longer hospital stay (both $p<0.05$ ).

The early postoperative complication rate was higher in the open repair group. Incision pain was the most common early complication, being the only early complication in the Lichtenstein group and comprising $5 \%$ of the 


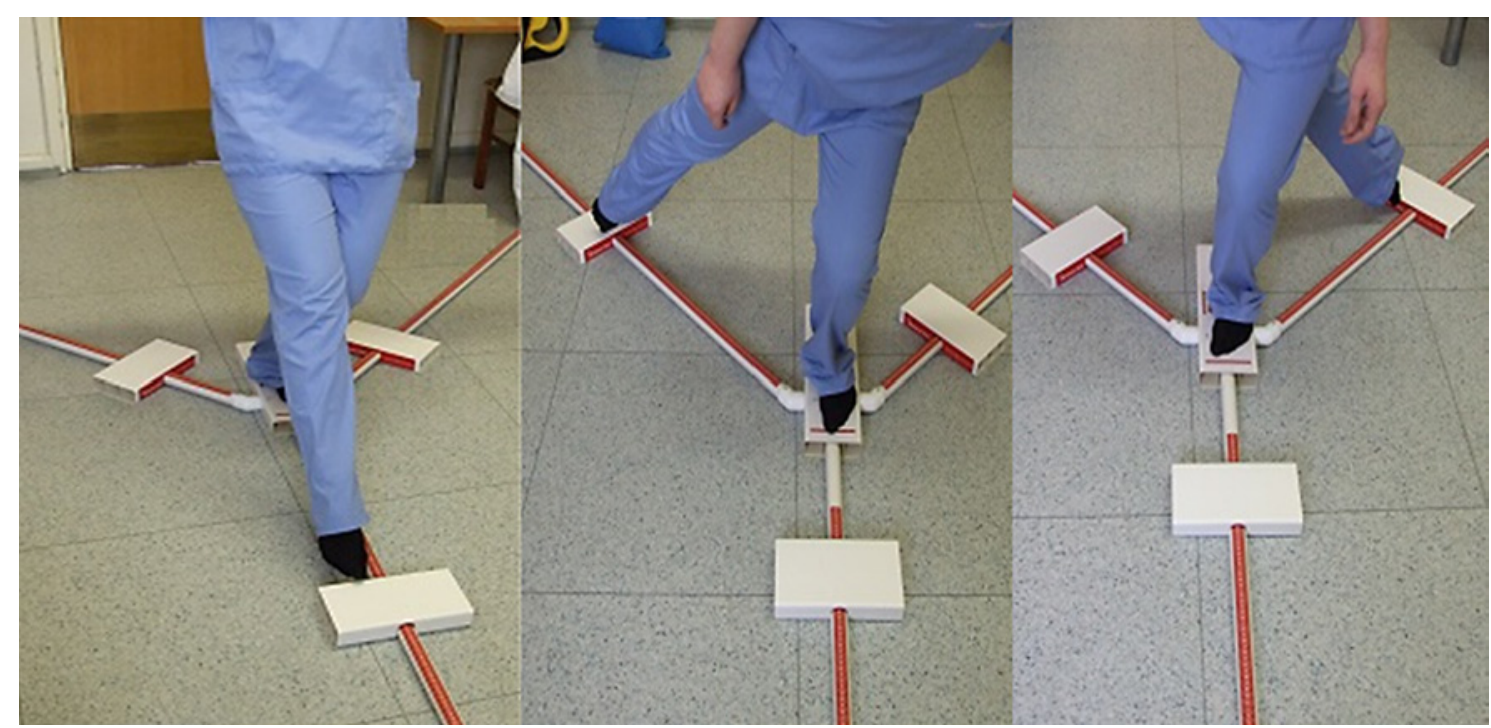

Fig. 7. Stability test: FMS Y Balance Test absolute reach distance.

Table 1. General characteristics

\begin{tabular}{llll}
\hline & Lichtenstein & Laparo-endo & $p$ value \\
\hline Age, years & $62 \pm 7$ & $56 \pm 11.6$ & $>0.05$ \\
BMI & & & $>0.05$ \\
$\quad<30$ & 62.5 & 81.8 & \\
$\quad 30-35$ & 37.5 & 18.2 & $>0.05$ \\
$\begin{array}{l}\text { Symptoms } \\
\quad \text { None }\end{array}$ & - & 8.3 & \\
$\quad$ Groin pain, tumor & 100 & 91.7 & \\
$\begin{array}{l}\text { Previous surgery } \\
\text { Hernia type }\end{array}$ & 50 & 16.7 & $>0.05$ \\
$\quad$ Direct & 14.3 & 21.1 & $>0.05$ \\
$\quad$ Indirect & 71.4 & 63.2 & \\
$\quad$ Combined & 14.3 & 15.8 & \\
$\begin{array}{l}\text { Surgery time, min } \\
\text { Hospital stay, days }\end{array}$ & $88 \pm 14.8$ & $71.3 \pm 27$ & $<0.05$ \\
& $2.9 \pm 2.21$ & $1.09 \pm 0.29$ & $<0.05$ \\
\hline
\end{tabular}

Values are presented as mean \pm SD or \%. Laparo-endo, laparoscopic-endoscopic hernia repair.

early complications in the laparoscopic-endoscopic repair group; however, in addition, $5 \%$ of the early complications were abdominal wall hematoma and $5 \%$ were recurrence in the minimally invasive repair group. The rate of late postoperative complications, such as abdominal wall or scrotum hematoma, was a little bit higher in the laparoscopic-endoscopic repair group (Table 2).

\section{Mobility Test}

The changes in adduction angle 1 day after surgery compared with the preoperative results were significantly smaller in the laparoscopic-endoscopic repair group ( $\mathrm{Ta}$ ble 3). After 2 weeks, the adduction angle had become normal in the minimally invasive hernia repair group.
There was still a significant difference in adduction angles 2 weeks after surgery in the Lichtenstein group compared with preoperative results. The adduction angle returned to normal ranges only after 4 weeks in the open hernia repair group.

The flexion angle changes were also significantly smaller in the minimally invasive hernia repair group on the first postoperative day. One week after surgery, the flexion angle values had reached the preoperative level in the laparoscopic-endoscopic hernia repair group and a significant difference was reached between the groups. As the adduction angle values, the flexion angle parameters became normal only 4 weeks after surgery in the Lichtenstein repair group.

The change in extension angle was also significantly smaller in the minimally invasive hernia repair group on the first postoperative day, and the extension angle became normal 1 week after surgery. In the Lichtenstein repair group, the extension angle became normal only 2 weeks after surgery.

\section{Strength Test}

The flexion strength changes were smaller in the Lichtenstein repair group on the first postoperative day (Table 4). One week after surgery, this difference became smaller and flexion strength became normal in the laparoscopic-endoscopic group 2 weeks after surgery. In the Lichtenstein repair group, flexion strength recovered 4 weeks after surgery.

The adduction strength changes were smaller in the laparoscopic-endoscopic repair group on the first postoperative day and 1 week after surgery. Adduction strength became normal 2 weeks after surgery in the laparoscopic-endoscopic repair group. Adduction strength 
Table 2. Postoperative complications

\begin{tabular}{llll}
\hline & Lichtenstein & Laparo-endo & $p$ value \\
\hline Early complications & & & $<0.05$ \\
$\quad$ None & 62.5 & 82.4 & \\
$\quad$ Inguinal pain & 37.5 & 5 & \\
Abdominal wall hematoma & - & 5 & \\
$\quad-\quad$ & 5 & $<0.05$ \\
Recurrence & & & \\
$\quad$ None complications & 87.7 & 73.9 & \\
Abdominal wall/scrotum hematoma & 12.5 & 26.1 & \\
\hline
\end{tabular}

Values are presented as \%. Laparo-endo, laparoscopic-endoscopic hernia repair.

Table 3. Mobility test changes

\begin{tabular}{|c|c|c|c|c|c|c|c|c|c|}
\hline & \multicolumn{3}{|l|}{ Flexion angle } & \multicolumn{3}{|c|}{ Extension angle } & \multicolumn{3}{|c|}{ Adduction angle } \\
\hline & Lichtenstein & Laparo-endo & $p$ value & Lichtenstein & Laparo-endo & $p$ value & Lichtenstein & Laparo-endo & $p$ value \\
\hline 1 day & $-16.41 \pm 6.58$ & $-6.53 \pm 14.14$ & 0.01 & $-4.83 \pm 3.81$ & $-1.16 \pm 1.63$ & 0.036 & $-16.83 \pm 6.08$ & $-2.79 \pm 10.24$ & 0.004 \\
\hline 1 week & $-9.25 \pm 4.59$ & $0.5 \pm 11.45$ & 0.036 & $-4.83 \pm 2.08$ & $2 \pm 3.94$ & 0.008 & $-8.08 \pm 5.32$ & $0.05 \pm 13.1$ & 0.224 \\
\hline 2 weeks & $-1.67 \pm 5.03$ & $0 \pm 9.75$ & 0.898 & $0.67 \pm 1.6$ & $1.2 \pm 4.95$ & 1 & $-3.58 \pm 6.93$ & $-0.61 \pm 11.26$ & 0.282 \\
\hline 4 weeks & $1.2 \pm 3.09$ & $6.25 \pm 6.22$ & 0.127 & $0.9 \pm 2.19$ & $2 \pm 4.34$ & 0.329 & $1.3 \pm 2.28$ & $4.68 \pm 16.96$ & 0.065 \\
\hline
\end{tabular}

Bold type denotes significance. Laparo-endo, laparoscopic-endoscopic hernia repair.

Table 4. Strength test changes

\begin{tabular}{|c|c|c|c|c|c|c|c|c|c|}
\hline & \multicolumn{3}{|c|}{ Flexion strength } & \multicolumn{3}{|c|}{ Adduction strength } & \multicolumn{3}{|c|}{ Abduction strength } \\
\hline & Lichtenstein & Laparo-endo & $p$ value & Lichtenstein & Laparo-endo & $p$ value & Lichtenstein & Laparo-endo & $p$ value \\
\hline 1 day & $-6.43 \pm 2.63$ & $-8.28 \pm 5.78$ & 0.473 & $-6.2 \pm 2.14$ & $-1.8 \pm 5.12$ & 0.06 & $-4.85 \pm 2.1$ & $-3.89 \pm 4.73$ & 0.516 \\
\hline 1 week & $-4.2 \pm 2.03$ & $-1.8 \pm 7.01$ & 0.345 & $-3.27 \pm 2.44$ & $1.1 \pm 6.1$ & 0.059 & $-3.76 \pm 2.54$ & $-1.65 \pm 2.84$ & 0.181 \\
\hline 2 weeks & $-2.39 \pm 3.57$ & $0.9 \pm 6.94$ & 0.323 & $-0.87 \pm 2.14$ & $1.84 \pm 5.01$ & 0.106 & $-0.5 \pm 0.89$ & $-0.96 \pm 1.82$ & 0.639 \\
\hline 4 weeks & $-0.18 \pm 1.33$ & $4.66 \pm 6.02$ & 0.149 & $0.06 \pm 0.58$ & $3.4 \pm 7.3$ & 0.435 & $-0.16 \pm 0.9$ & $1.93 \pm 2.8$ & 0.222 \\
\hline
\end{tabular}

Laparo-endo, laparoscopic-endoscopic hernia repair.

recovered only 4 weeks after surgery in the Lichtenstein repair group.

The changes in abduction strength were slightly smaller in the endoscopic-laparoscopic repair group on the first postoperative day. A similar difference in change between the groups remained 1 week after surgery. The abduction strength changes were smaller without any significant difference in the Lichtenstein group 2 weeks after surgery. Abduction strength fully recovered 4 weeks after surgery in the laparoscopic-endoscopic repair group, but some changes still occurred in the Lichtenstein repair group.

\section{Stability Test}

The stability test changes were lower in the laparoscopic-endoscopic repair group on the first postoperative day, and these results became normal 1 week after surgery
(Table 5). The stability test results became normal only 4 weeks after Lichtenstein repair.

\section{Discussion}

Hernia repair not only affects the individual patient but also has a significant socioeconomic relevance and an important impact on the costs for the health care system [8]. This is why the ideal technique for inguinal hernia repair should cause as few complications and little postoperative pain as possible but lead to fast recovery and return to normal activity [4]. Although open tension release has been a widely used routine technique for a long time, laparoscopic-endoscopic techniques should be considered more effective. As many studies have shown, there is no significant difference in intraoperative and 
Table 5. Stability test changes in FMS Y Balance Test absolute reach distance

\begin{tabular}{lccl}
\hline \multirow{4}{*}{} & \multicolumn{2}{l}{ FMS Y Balance Test } \\
\cline { 2 - 4 } & Lichtenstein & Laparo-endo & $p$ value \\
\hline 1 day & $-7.39 \pm 9.94$ & $-3.01 \pm 14.16$ & 0.154 \\
1 week & $-2.97 \pm 6.98$ & $5.16 \pm 13.84$ & 0.228 \\
2 weeks & $-1.06 \pm 6.64$ & $5.79 \pm 13.35$ & 0.151 \\
4 weeks & $2.73 \pm 5.88$ & $6.92 \pm 9.78$ & 0.268 \\
\hline
\end{tabular}

Laparo-endo, laparoscopic-endoscopic hernia repair.

postoperative complications and recurrence rates, and laparoscopic-endoscopic techniques are superior in that they cause less chronic, acute, and late postoperative pain and have a faster recovery time.

The results of our study show that hip and leg mobility recovers faster after endoscopic hernia repair. Although we had significant differences in only few pelvic function test groups, because of the small size of the study group, we can observe a tendency that hip and leg mobility, strength, and stability recover faster after laparoscopic-endoscopic hernia repair. Almost all changes in function tests between preoperative results and outcomes 1 day after surgery, except in flexion strength, were smaller after laparoscopic-endoscopic procedures. Most of the hip and leg functions, except flexion and extension strength, had fully recovered by 1 week after laparoscopic-endoscopic hernia repair. Only extension strength was slightly lower 2 weeks after surgery compared with preoperative results. All functions were at the same level or better 4 weeks after laparoscopic-endoscopic hernia repair. Recovery after Lichtenstein hernia repair took about 4 weeks or longer, apart from the extension angle, which became normal on the second postoperative week. Thus, all hip and leg functions of the patients who underwent laparoscopic-endoscopic hernia repair had recovered 2 or 3 weeks earlier than after Lichtenstein surgery.

Like other studies, our study did not show any significant differences in early and late postoperative complications and recurrence rates. However, the early complication rate was slightly higher after Lichtenstein hernia repair, and there was only a slightly lower rate of late postoperative complications in the same group, without any significant difference. Unfortunately, we had 1 case of early recurrence on the first postoperative day after total extraperitoneal hernia repair due to large (type 3 ) direct inguinal hernia. The patient was reoperated on the next day, and the mesh was dislocated. Transabdominal preperitoneal hernia repair with mesh fixation was performed. In addition, the surgery time and hospital stay were significantly shorter in the laparoscopic-endoscopic hernia repair group.
The main strength of our study is that we assessed the recovery time after inguinal hernia surgery using not only conditional subjective scales, but also standardized physical tests of hip and leg function. We admit that this study was nonrandomized and had a low number of patients in both groups, but it was a pilot study to verify if selected hip and leg mobility, stability, and strength tests were suitable for the evaluation of recovery after inguinal hernia repair.

As mentioned before, all the studies investigating recovery after inguinal hernia repair have used a visual analog scale, a short-form survey instrument, or patients' return-to-normal-activity time, and there is a lack of studies on objective pelvic functional recovery [4-7]. Some randomized controlled trials - which used a visual analog scale, recorded the use of analgesic drugs, and assessed activities of daily living with various health survey questionnaires - showed that visual analog pain scores after surgery were lower and patients returned to work sooner after laparoscopic repair; also the difference in the rates of recurrence between the two groups was similar $(p=0.05)[5,8]$. Similar results were obtained by comparing TEP with Lichtenstein hernia repair: no significant difference was identified in recurrence rates between the TEP and the Lichtenstein technique, and TEP repair was found to have benefits compared with Lichtenstein repair as regards postoperative complication rates, pain at rest, and pain on exertion [6]. In our clinical study, we used objective hip and leg mobility, strength, and stability tests which are discussed among physiotherapists and also demonstrated that postoperative functional hip and leg recovery and return to normal activity were faster after laparoscopic-endoscopic hernia repair. There is some clinical research in which these tests were used to evaluate recovery of hip function after hip arthroplasty or sports traumas, but they have never been used to estimate hip and leg function after inguinal hernia repair $[9,10]$.

The results of our study provide more solid arguments, such as faster hip and leg mobility, strength, and stability recovery, for surgeons to choose a minimally invasive surgery technique for inguinal hernia repair.

As we consider these tests an acceptable method for the assessment of hip and leg functional recovery after inguinal hernia repair, a randomized trial with study power calculation should be performed. Also, there are no recommendations as to which endoscopic method, TAPP or TEP repair, is superior; thus, a similar study could be done to objectively compare endoscopic and laparoscopic surgical techniques. Of course, a smaller difference in postoperative changes between TAPP and TEP groups is expected, so the study size should be greater to obtain the best result. 


\section{Conclusions}

Firstly, by obtaining differences in hip and leg functional recovery between laparoscopic-endoscopic and Lichtenstein hernia repair methods, our hip and leg mobility, strength, and stability tests are useful to evaluate the recovery time after inguinal hernia repair. Also, these tests could be used as an objective tool for estimating recovery after the application of other inguinal hernia repair techniques.

Secondly, our study shows that hip and leg mobility, strength, and stability recover faster after minimally invasive inguinal hernia repair. In addition, there is no significant difference in early and late postoperative complications or recurrence rates between the groups.

\section{Statement of Ethics}

Informed consent was obtained from the patients for being included in this study. The study was conducted with approval from the Ethics Committee of the Lithuanian University of Health Sciences (protocol No. BEC-LSMUC(R)-26).

\section{Disclosure Statement}

All authors declare that there is no conflict of interest.

\section{Author Contributions}

All authors contributed extensively to the work presented in this paper, contributed to the writing of the manuscript, read and approved the final manuscript.

\section{References}

1 Kulacoglu H. Current options in inguinal hernia repair in adult patients. Hippokratia. 2011 Jul;15(3):223-31.

2 HerniaSurge Group. International guidelines for groin hernia management. Hernia. 2018 Feb;22(1):1-165.

3 Simons MP, Aufenacker T, Bay-Nielsen M, Bouillot JL, Campanelli G, Conze J, et al. European Hernia Society guidelines on the treatment of inguinal hernia in adult patients. Hernia. 2009 Aug;13(4):343-403.

4 Hamza Y, Gabr E, Hammadi H, Khalil R. Four-arm randomized trial comparing laparoscopic and open hernia repairs. Int J Surg. 2010;8(1):25-8
5 Liem MS, van der Graaf Y, van Steensel CJ, Boelhouwer RU, Clevers GJ, Meijer WS, et al. Comparison of conventional anterior surgery and laparoscopic surgery for inguinal-hernia repair. N Engl J Med. 1997 May;336(22): 1541-7.

6 Ahmed I, Dian A, Azam UF, Khan M. Evaluation of outcome of totally extra peritoneal laparoscopic inguinal hernia repair with Lichtenstein open repair. Pak Armed Forces Med J. 2015;65(1):16-21.

7 Srsen D, Druzijanić N, Pogorelić Z, Perko Z, Juricić J, Kraljević D, et al. Quality of life analysis after open and laparoscopic inguinal hernia repair - retrospective study. Hepatogastroenterology. 2008 Nov-Dec;55(88):2112-5.
8 Schmedt CG, Sauerland S, Bittner R. Comparison of endoscopic procedures vs Lichtenstein and other open mesh techniques for inguinal hernia repair: a meta-analysis of randomized controlled trials. Surg Endosc. 2005 Feb;19(2):188-99.

9 Sicard-Rosenbaum L, Light KE, Behrman AL. Gait, lower extremity strength, and selfassessed mobility after hip arthroplasty. J Gerontol A Biol Sci Med Sci. 2002 Jan; 57(1):M47-51.

10 Gonell AC, Romero JA, Soler LM. Relationship between the Y Balance Test scores and soft tissue injury incidence in a soccer team. Int J Sports Phys Ther. 2015 Dec;10(7):95566. 